\title{
Rare Endobronchial Polyps due to SARS-CoV-2 Infection
}

\section{Raghavendra Sanivarapu, MD*, James Kang, MD, Javed Iqbal, MD and Fatima Anjum, MD}

Division of Pulmonary \& Critical Care Medicine, Department of Internal Medicine, Nassau University Medical Center, USA

*Corresponding author: Raghavendra Sanivarapu, MD, Division of Pulmonary \& Critical Care Medicine, Department of Internal Medicine, Nassau University Medical Center, 2201 Hempstead Tn Pk, East Meadow, NY-11554, USA, Tel: 9172025654

\section{Introduction}

Since its discovery in December 2019, the Coronavirus disease 2019 (COVID-19) has spread to more than 70 countries around the world. The United States alone has seen more than 1.5 million cases, with more than 100,000 deaths to date [1]. The most common presenting symptoms are fever, cough and shortness of breath with median incubation periods of 5 days [2]. Severe symptoms can develop as a result of the aggressive inflammatory response. The role of bronchoscopy has been limited in severe cases and results are infrequently reported. We report a case of a young male with severe COVID-19 who developed multiple endobronchial polyps in the tracheobronchial tree seen on bronchoscopy examination.

\section{Case Report}

A 29-year-old male with no significant past medical history presented with cough, chills, subjective fevers, fatigue, body aches and shortness of breath for oneweek duration. Vitals on admission were BP: 124/74 mmHg, HR: 100 bpm, RR: 20/min, Temp: $97.6^{\circ} \mathrm{F}$. Chest $\mathrm{X}$-ray (CXR) revealed bilateral infiltrates. SARS-CoV-2 polymerase chain reaction test was positive. His condition rapidly deteriorated requiring intubation for acute hypoxemic respiratory failure. Analysis of repeat CXR, arterial blood gas and clinical course was consistent with severe acute respiratory distress syndrome. His hospital course was complicated by septic shock and femoral Deep Vein Thrombosis (DVT). The patient received ceftriaxone, azithromycin, hydroxychloroquine and enoxaparin. After 2.5 weeks since admission his CXR showed worsening infiltrates and a bronchoscopy was performed which showed erythematous mucosa only with mucopurulent material. The patient then developed cytokine storm with high grade fever of 105.9 ${ }^{\circ} \mathrm{F}$ and C-reactive protein of $15.3 \mathrm{mg} / \mathrm{dl}$. His serum IL-6 level was $540 \mathrm{pg} / \mathrm{ml}$ and was given tocilizumab. He also received convalescent plasma. A repeat bronchoscopy was performed a week later due to thick secretions and atelectasis showed friable broad-based endobronchial polyps throughout the tracheobronchial tree (Figure 1). Biopsy of lesions was not obtained as patient was high risk for bleeding due to full dose anticoagulation for DVT. The patient was given methylprednisolone for 7-day duration. Eventually patient was stabilized and transitioned to a tracheostomy for continued ventilator support. A repeat bronchoscopy was performed 5 weeks after admission, showing reduction in number of polyps and resolution at multiple sites (Figure 2). The patient was later transferred to a step-down unit where he continued to improve and was finally decannulated and discharged home after 10 weeks of hospitalization.

\section{Discussion}

Bronchoscopy examination of COVID-19 pneumonia has not been well documented in literature. Current guidelines from American Association for Bronchology and Interventional Pulmonology (AABIP) for COVID-19 are not supportive of bronchoscopy due to its limited usefulness in this disease process and potential for increased aerosol exposure to health-care workers $[3,4]$. The few documented COVID-19 bronchoscopy results revealed only mucosal swelling, mucopurulent secretions and congestion [5]. WHO/IASLC classifies inflammatory endobronchial polyps as a distinct type of non-neoplastic tumors of lung [6]. Although endo-

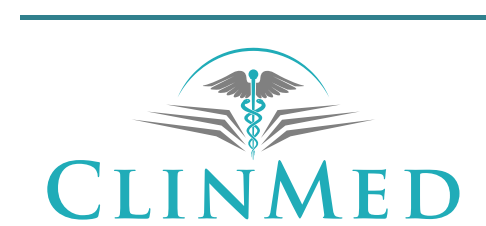

INTERNATIONAL LIBRARY

Citation: Sanivarapu R, Kang J, Iqbal J, Anjum F (2020) Rare Endobronchial Polyps due to SARS-CoV-2 Infection. Int J Respir Pulm Med 7:142. doi.org/10.23937/2378-3516/1410142

Accepted: September 22, 2020; Published: September 24, 2020

Copyright: (C) 2020 Sanivarapu R, et al. This is an open-access article distributed under the terms of the Creative Commons Attribution License, which permits unrestricted use, distribution, and reproduction in any medium, provided the original author and source are credited 


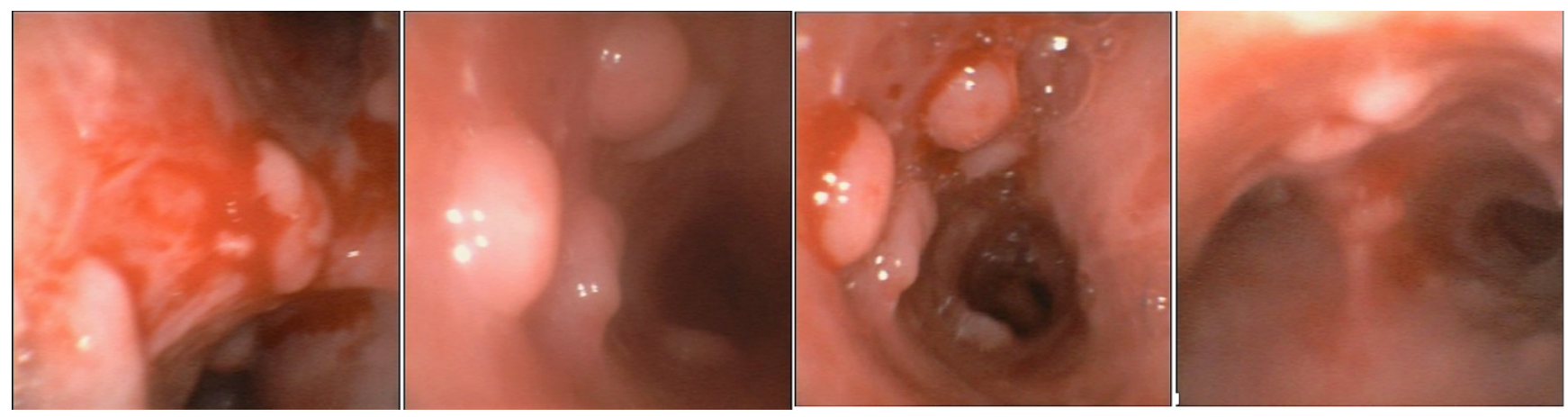

Figure 1: Bronchoscopy showing multiple endobronchial polyps.

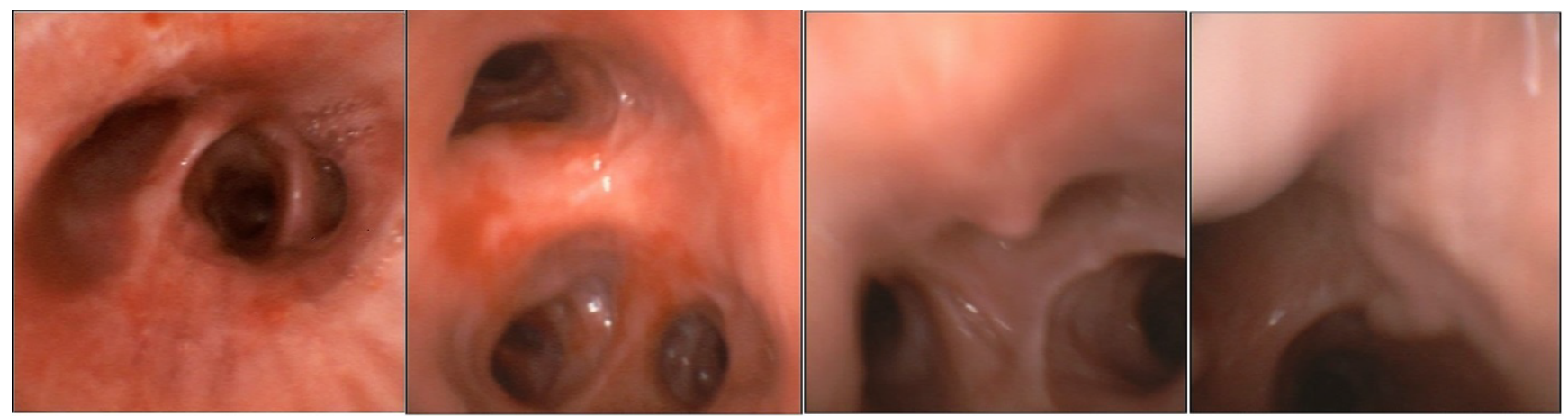

Figure 2: Resolution of endobronchial polyps on repeat bronchoscopy.

bronchial polyps have been reported in some inflammatory conditions for example bacterial infections and malignancy [7-9], we are reporting the first case of endobronchial polyps related to SARS-CoV-2 infection. A case report by Adams, et al. showed multiple polyps post thermal inhalation injury which resolved after steroid course strongly indicating inflammation as primary cause. The mechanism behind the COVID-19 pathogenesis is thought to be a result of a severe inflammatory response implicating progressive multi-organ damage [10]. Our patient did not have these endobronchial polyps on initial bronchoscopy; their development strongly suggests COVID-19 as the primary insult through inflammatory mechanisms [11-13].

\section{Conclusion}

While bronchoscopy has not played a large role during this pandemic, it should be considered to further enhance our understanding of this disease process.

\section{References}

1. (2020) United States covid-19 cases and deaths by state.

2. Lauer SA, Grantzm KH, Bi Q, Jones FK, Zheng Q, et al (2020) The incubation period of coronavirus disease 2019 (COVID-19) from publicly reported confirmed cases: estimation and application. Ann Intern Med 172: 577-582.

3. Mehta P, McAuley DF, Brown M, Sanchez E, Tattersall RS, et al. (2020) COVID-19: Consider cytokine storm syndromes and immunosuppression. Lancet 395: 1033-1034.

4. Wang C, Xie J, Zhao L, Fei X, Zhang H, et al. (2020) Aveolar macrophage activation and cytokine storm in the pathogenesis of severe COVID-19. Immunology.
5. Sanders JM, Monogue ML, Jodlowski TZ, Cutrell JB (2020) Pharmacologic treatments for coronavirus disease 2019 (COVID-19): A review. JAMA 323: 1824-1836.

6. Murayama T, Amitani R, Tsuyuguchi K, Watanabe I, Kimoto T, et al. (1998) Polypoid bronchial lesions due to Scedosporium apiospermum in a patient with Mycobacterium avium complex pulmonary disease. Eur Respir J 12: 745-747.

7. Ming Li, Guoliang Zhang, Aimei Peng, Changhui Wang (2012) Bronchial fibroepithelial polyp: A case report and review of the literature. Intern Med 52: 373-376.

8. Pollefliet C, Peters K, Janssens A, Luijks A, Van Bouwel E, et al. (2009) Endobronchial lipomas: Rare benign lung tumors, two case reports. J Thorac Oncol 4: 658-660.

9. Takeuchi A, Kanemitsu Y, Takakuwa O, Ito K, Kitamura Y, et al. (2018) A suspected case of inflammatory bronchial polyp induced by bronchial thermoplasty but resolved spontaneously. J Thorac Dis 10: 678-681.

10. Xu X, Han M, Li T, Sun W, Wang D, et al. (2020) Effective treatment of severe COVID-19 patients with tocilizumab. Proc Natl Acad Sci 117: 10970-10975.

11. (2020) Handbook of COVID-19 Prevention and Treatment.

12. Bhatraju PK, Ghassemieh BJ, Nichols M, Kim R, Jerome $\mathrm{KR}$, et al. (2020) Covid-19 in critically ill patients in the Seattle region-case series. N Engl J Med 382: 2012-2022.

13. Tay MZ, Poh CM, Rénia L, MacAry PA, Ng LF (2020) The trinity of COVID-19: Immunity, inflammation and intervention. Nat Rev Immunol 20: 363-374. 